\title{
PTEN Genetic and Epigenetic Alterations Define Distinct Subgroups in North Indian Breast Cancer Patients
}

\author{
Zakia Kazim ${ }^{1}$, Khushnuma Wahabi ${ }^{1}$, Ahmad Perwez ${ }^{1}$, Pawanindra Lal ${ }^{2}$, \\ Moshahid Alam Rizvi ${ }^{\text {** }}$
}

\begin{abstract}
Background: Breast cancer is a heterogeneous disease that can be subdivided on the basis of histopathological features, genetic alterations, and gene-expression profiles. PTEN gene is considered an established tumor suppressor gene in different types of cancer including breast cancer. However, the role of $P T E N$ alterations in north Indian breast cancer has not been explored especially in defining a group with distinct histological factors. Methodology: 181 sporadic breast cancer and their adjacent normal tissues were included in the present study. We analyzed methylation and LOH through MS-PCR and microsatellite markers respectively. While, for PTEN protein expression, we used immunohistochemistry. All the molecular findings were correlated with the clinicopathological parameters of the patients to underline clinical relevance. Results: We found that LOH and methylation of the PTEN promoter were significantly associated with loss of PTEN protein expression, while, PTEN mutation was a rare event. Furthermore, out of 46 double hit cases (i.e., having both methylation and $\mathrm{LOH}), 70 \%(32 / 46)$ cases showed complete loss of PTEN expression $(\mathrm{P}=0.0249)$. Both $\mathrm{LOH}$ and $P T E N$ promoter methylation were associated significantly with age and clinical stage, while, methylation and loss of PTEN expression were associated with high grade and Her-2 negativity. In addition, a quadruple (ER/PR/ Her-2 and PTEN) negative group with distinct features was found. Conclusion: The pattern of PTEN expression and its correlation with the clinical parameters indicates that loss of PTEN expression defines a clinical group with distinct features. Hence, PTEN expression provides differential therapeutic strategies for north Indian breast cancer.
\end{abstract}

Keywords: PTEN-Indian breast cancer- expression- methylation- LOH

Asian Pac J Cancer Prev, 20 (1), 269-276

\section{Introduction}

Breast cancer is the most frequent cause of cancer-related death among women worldwide, claiming more than 400,000 lives every year (Jemal et al., 2011). In India, a rising trend in breast cancer incidence is observed, which is predicted to $31 \%$ rise by 2020 (Anonymous, 2009). Breast cancer is a biologically and clinically heterogeneous disease where gene/pathway function changes with specific genetic and epigenetic alterations (Hanahan and Weinberg, 2011). Thus, understanding the contribution of gene expression in profiling the clinical subgroups and their assessment as prognostic factors are of great importance in the prediction of the disease.

PTEN (Phosphatase and tensin homolog) gene, located on chromosome 10 is a well-known tumor suppressor gene, which is frequently altered in a wide spectrum of cancers (Li et al., 1997; Steck et al., 1997). It is a lipid phosphatase, which negatively regulates PI3K-AKT pathway, thereby regulating a plethora of various cellular processes like cell cycle, apoptosis, cell survival, cell growth, adhesion, migration and spreading (Vivanco and
Sawyers, 2002; Lu et al., 1999; Tamura et al., 1998). PTEN expression and function are usually altered in cancer by genetic and epigenetic alterations. PTEN has been found to be linked with the breast cancer initiation and progression through a number of mechanisms such as mutation, loss of heterozygosity at the PTEN gene locus, epigenetic silencing of PTEN promoter and posttranslational modifications (Song et al., 2012; Salmenaet al., 2008). Recently, PTEN alteration was found to be associated with an emerging tumor suppressor gene, Parkin, which opened a new horizon to its regulation (Wahabiet al., 2018). In case of breast cancer, structural mutations of the PTEN gene are very rare while familial cancer syndrome known as Cowden disease has $25 \%$ to $50 \%$ lifetime risk of developing breast cancer (Nelen et al., 1999; Lynch et al., 1997). Loss of heterozygosity (LOH) at PTEN (10q23) locus is approximately 30-40\% (Singh et al., 1998). Additionally, PTEN promoter methylation has been suggested as one of the most common molecular alterations involved in the pathogenesis of various malignancies including breast cancer (Shetty et al., 2011; Zhang et al., 2013). 
Molecular classifications are indicators of genetic tumor heterogeneity, which can lead to improved stratification of cancer into low- and high-risk groups for personalized therapy. Despite several types of research conducted globally on PTEN gene implications in breast cancer, the exact mechanism of altered PTEN involvement and its association with clinical variables in breast cancer is not well established in Indian patients. Keeping the significance of the PTEN gene in breast cancer initiation and prognosis, in view, the present study aims to evaluate the PTEN gene abnormalities and its association with distinct clinical parameters in north Indian breast cancer patients.

\section{Materials and Methods}

\section{Ethics Statement}

The present study was approved by the Institutional Ethics Committee (IEC) of Jamia Millia Islamia, New Delhi. A written informed consent as approved by the IEC was obtained from all the participants of the study.

\section{Breast tissue samples and DNA extraction}

A total of one hundred and eighty one $(n=181)$ invasive breast carcinoma samples along with their adjacent noncancerous tissues were collected after mastectomy from LNJP Hospital, New Delhi, along with their associated clinicopathological parameters. Biopsies were divided into two parts; one part was placed in a Phosphate saline buffer (PBS; $\mathrm{pH}=7.2$ ) and stored as soon as possible in $-80^{\circ} \mathrm{C}$ for DNA extraction. The other part was collected in $10 \%$ formalin for histopathological investigations. Genomic DNA was extracted by using ZR Genomic DNATM MiniPrep Kit (Zymo research, USA) according to manufacturer's protocol. The quantity and quality of the DNA were determined by the Nanodrop (Thermo Fisher Scientific, USA) Spectrophotometer and further by running $1 \%$ agarose gel stained with ethidium bromide.

\section{PTEN allelotyping; estimation of heterozygosity}

DNA from tumor and non-tumor samples was amplified using three published polymorphic microsatellite markers located within and close to the PTEN gene to detect loss of heterozygosity (LOH). Markers D10S215 and D10S583 are located within the PTEN gene, while, the D10S541 marker is found at the flanking region. Briefly, PCR reactions were performed in $25 \mu \mathrm{l}$ volumes with a final concentration of $200 \mu \mathrm{M}$ dNTP, $1.5 \mathrm{mM} \mathrm{MgCl} 2,10 \mathrm{mM}$ Tris- $\mathrm{HCl}(\mathrm{pH} 8.3), 50 \mathrm{mM} \mathrm{KCl}, 0.4 \mu \mathrm{M}$ of each primer, 0.5 units of Taq DNA polymerase (BangloGenei, India) and $60 \mathrm{ng}$ of DNA template. The sequence of the primers is shown in supplementary table1. PCR was done and the products were resolved (Figure 1) as described previously (Rizvi et al., 2012).

\section{PCR-SSCP analysis}

Isolated genomic DNAs were subjected to PCR-SSCP for mutational analysis. The primers and conditions used for the different PTEN exons were as described in an earlier study (Rizvi et al., 2011). The PCR products were resolved in 2\% agarose gels and visualized by staining with ethidium bromide. Non-radioisotopic SSCP analysis was performed as previously described (Rizvi et al., 2011). After electrophoresis, silver staining was done for visualization. PCR products that revealed mobility shifts on SSCP analysis were sequenced for mutation.

\section{Bisulfite modification of DNA and MS-PCR}

Genomic DNA from breast cancer and normal specimens were modified by treatment with sodium bisulfite using EZ DNA Methylation-Gold kit (Zymo Research, USA) according to manufacturer's protocol. Two separate sets of primers were used for the amplification of the promoter region of the $P T E N$ gene, one primer specific for unmethylated sequence 5'GTGTTGGTGGAGGTAG TTGTTT-3'(sense) and 5'- CCACTTAACTCTAAACC ACAACCA-3'(antisense) was used to amplify a $162 \mathrm{bp}$ product, while the other primer specific for methylated sequence 5'-TTCGTTCGTCGTCGTCGTATTT-3'(sense) and 5'-GCCGCTTAACTCTAAACCGCAACCG-3'(ant isense) was used to amplify 206-bp product. Completely methylated and unmethylated human genomic DNA (Zymo Research Corp., Orange, CA) were used as positive control for the methylated allele (Figure 2).

\section{PTEN Immunohistochemistry}

Paraffin-embedded blocks were made from formalin-fixed tissues. The sectioning was done by microtome and fixed on glass slides. The whole procedure was followed as mentioned in an earlier study (Ali et al., 2014) using anti-PTEN (Thermofisher) antibody (1:100). Afterward, slides were incubated with biotinylated horse anti-mouse IgG followed by avidin-peroxidase. The reaction was then visualized with diaminobenzidine (DAB). Hematoxylin was used as a counterstain. Each run included appropriate positive and negative control slides. Representative pictures are shown in Figure 3.

\section{Interpretation of staining}

Slides were evaluated by an expert histopathologist under a light microscope at 400X magnification (Figure 3 ). Stained slides were graded as negative (0), low expression (up to $25 \%$ ), moderate expression (up to $50 \%$ ), and high expression (more than 50\%). Furthermore, if the expression of a protein was more than $5 \%$ only then it was considered a positive case.

\section{Statistical analysis}

All the statistical comparisons were performed with Fisher's exact test for significance. P-values $\mathrm{p}<0.05$ were accepted as statistically significant.

\section{Results}

PTEN allelotyping and correlation with clinical parameters of breast cancer

Three markers viz D10S215, D10S583, and D10S541 (Table.1) were used to investigate $\mathrm{LOH}$ at $10 \mathrm{q} 23$ region. $54 \%(98 / 181)$ cases showed loss of heterozygosity at the $10 \mathrm{q}$ region. The highest rate of $\mathrm{LOH}(31 \%)$ was observed at intragenic marker D10S583 when compared with either loci within (D10S215) gene (26\%) or flanking region of 
Table 1. Correlation of the PTEN Protein Expression with LOH and Promoter Methylation

\begin{tabular}{|c|c|c|c|c|c|c|}
\hline & \multirow[t]{2}{*}{ Total (Cases) } & \multicolumn{4}{|c|}{ PTEN Expression } & \multirow[t]{2}{*}{$P$ value } \\
\hline & & Negative & Low & Moderate & High & \\
\hline \multicolumn{7}{|l|}{ PTEN gene } \\
\hline Without LOH & $83(46)$ & $35(42)$ & $16(19)$ & $14(17)$ & $18(22)$ & \multirow[t]{3}{*}{$<0.0001$} \\
\hline With $\mathrm{LOH}$ & $98(54)$ & $62(63)$ & $33(34)$ & 3 (3) & $0(0.0)$ & \\
\hline Total & $181(100)$ & $97(54)$ & $49(27)$ & $17(9)$ & $18(10)$ & \\
\hline \multicolumn{7}{|l|}{ PTEN promoter } \\
\hline Unmethylated & $87(48)$ & $38(44)$ & $25(29)$ & $7(8)$ & $17(20)$ & \multirow[t]{3}{*}{0.002} \\
\hline Methylated & $94(52)$ & $59(63)$ & $24(26)$ & $10(11)$ & $1(1)$ & \\
\hline Total & $181(100)$ & $97(54)$ & $49(27)$ & $17(9)$ & $18(10)$ & \\
\hline
\end{tabular}

the gene $(23 \%)$ as summarized in Figure $1 . \mathrm{LOH}$ was significantly associated with age $[\mathrm{P}=0.0018,<50(65 \%)$ vs $\geq 50(42 \%)$ cases], advanced clinical stage $[\mathrm{P}<0.0001$, stage III+IV (81\%) vs stage I+II (38\%)] and ER positivity $[\mathrm{P}=0.0197$, ER positive $(75 \%)$ vs ER negative $(50 \%)$ cases] (Supplementary Table 2).

PTEN promoter methylation and correlation with clinical parameters of breast cancer

Overall, we found 51.9\% (94/181) PTEN promoter methylation. $P T E N$ promoter methylation was significantly correlated with advancing age $[\mathrm{P}<0.0001,>50(79 \%)$ vs
$<50(27 \%)$ cases], Clinical stage $[\mathrm{P}=0.0001$, stage III + IV (71\%) vs stage I+II (40\%)] advanced histological grade $[\mathrm{P}=0.0001$, grade III $+\mathrm{IV}(74 \%)$ vs grade $\mathrm{I}+\mathrm{II}(42 \%)]$ and Her-2 negative status $[\mathrm{P}=0.003$, Her 2 negative $(69 \%)$ vs Her2 positive ( $45 \%$ ) cases] as described in Supplementary Table 3 .

PTEN expression and correlation with clinical parameters of breast cancer

Out of 181 breast cancer tissue samples 54\% (97/181) cases showed loss of PTEN protein expression. Loss of $P T E N$ expression was significantly correlated with post-
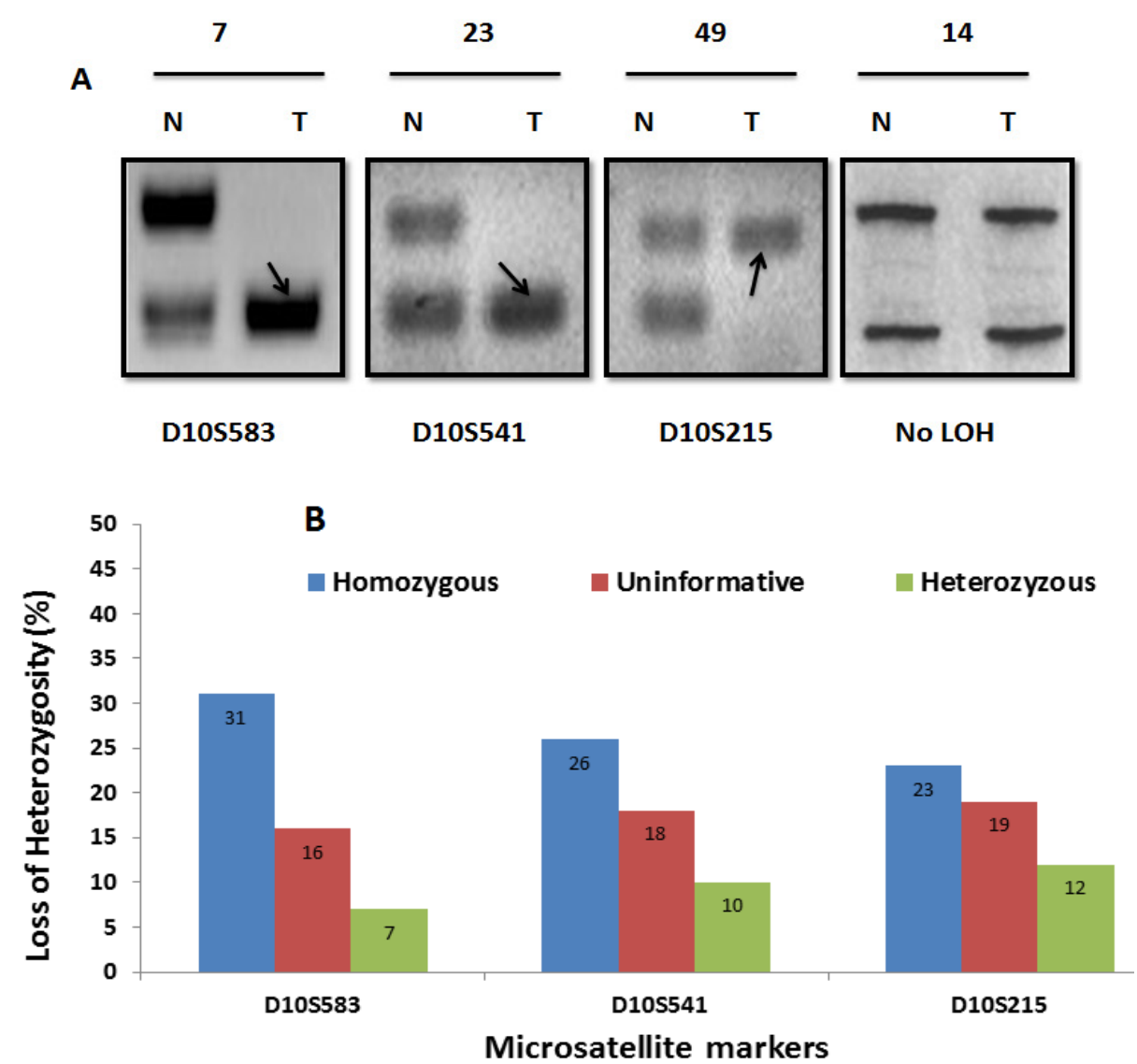

Figure 1. Loss of Heterozygosity (LOH) at Each of the Three Loci of 10q23 Region in Breast Cancer. (A) Silverstained polyacrylamide gel containing amplified product from the normal mucosa $(\mathrm{N})$ and tumor $(\mathrm{T})$ of patients (identified by numbers) with breast cancer. The tumors from patients 7, 23 and 49 showing loss of heterozygosity at different loci studied in the PTEN gene (arrowhead) while, patient 14 did not show the LOH and patient 53 was uninformative. All photographs are cropped and inverted to a white background for clarity. (B) Bar diagram showing the comparison of frequencies of $\mathrm{LOH}$ detected at three microsatellite markers in tumor samples from patients with breast cancer. 


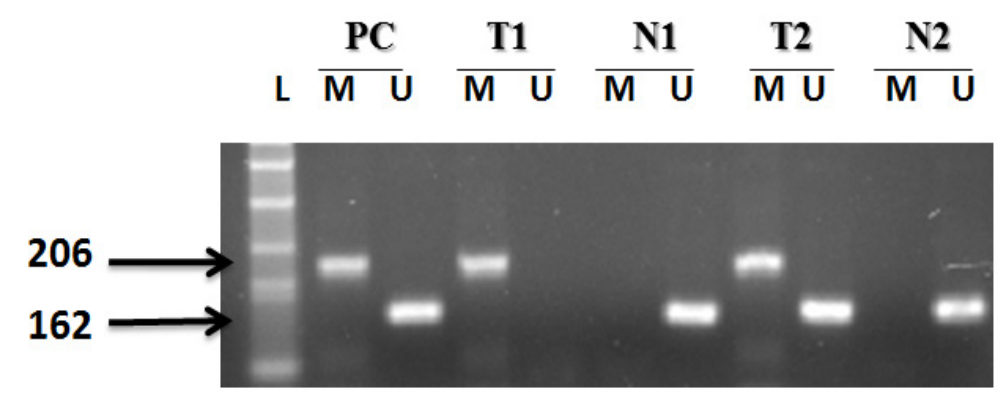

Figure 2. MS-PCR Gel Picture, Representing Promoter Hypermethylation of PTEN Gene in Breast Cancer. The lanes T1 to N2 correspond to DNA from patients with breast cancer; L, Ladder 100bp; U, Unmethylated PTEN promoter (product size $162 \mathrm{bp}$ ); M, Methylated PTEN promoter (product size-206 bp); PC, positive control for unmethylated and methylated alleles (completely unmethylated and methylated DNA controls, respectively); N, normal tissue sample; T, tumor tissue sample.

menopausal status $[\mathrm{P}<0.0001$, postmenopausal $(68 \%)$ vs premenopausal (33\%) cases], Clinical stage $[\mathrm{P}=0.0086$, stage I+II (56\%) vs stage III+IV (49\%)], advanced histological grade $[\mathrm{P}=0.0081$, grade III + IV $(71.9 \%)$ vs grade I+II (45\%)], Her-2 negative status $[\mathrm{P}<0.0001$, Her2 negative (77\%) vs Her 2 positive (44\%) cases] and triple negative cases $[\mathrm{P}=0.0043$, TNBC $(82 \%)$ vs TPBC (57\%) cases] as shown in Supplementary Table 4.

PTEN allelotyping and correlation with PTEN protein expression of breast cancer

In the present study, 54\% (98/181) breast cancer cases showed $\mathrm{LOH}$ in the $10 \mathrm{q} 23$ region. On the other hand, 54\% (97/181) cases have shown loss of PTEN

Table 2. Correlation Analysis of PTEN Protein Expression and Methylation in Samples Having Methylated PTEN Promoter or PTEN Loss in Indian Breast Cancer

\begin{tabular}{|c|c|c|c|c|c|c|c|c|}
\hline \multirow[t]{2}{*}{ Clinical Parameters } & \multirow{2}{*}{$\begin{array}{c}\text { Total } \\
(\mathrm{N}=73)\end{array}$} & \multicolumn{2}{|c|}{ PTEN Methylated } & \multirow[t]{2}{*}{ P-value } & \multirow{2}{*}{$\begin{array}{c}\text { Total } \\
(\mathrm{N}=108)\end{array}$} & \multicolumn{2}{|c|}{ PTEN loss } & \multirow[t]{2}{*}{ p-value } \\
\hline & & PTEN absent & PTEN present & & & $\begin{array}{c}\text { Unmethylated } \\
\text { PTEN }\end{array}$ & $\begin{array}{l}\text { Methylated } \\
\text { PTEN }\end{array}$ & \\
\hline \multicolumn{9}{|l|}{ Age } \\
\hline$<50$ & 48 & $27(56)$ & $27(56)$ & 0.206 & 47 & $20(43)$ & $27(57)$ & 0.539 \\
\hline$\geq 50$ & 46 & $32(70)$ & $32(70)$ & & 50 & $18(36)$ & $32(64)$ & \\
\hline \multicolumn{9}{|l|}{ Menopausal Status } \\
\hline Premenopausal & 34 & $14(41)$ & $14(41)$ & 0.002 & 24 & $10(42)$ & $14(58)$ & 0.812 \\
\hline Postmenopausal & 60 & $45(75)$ & $45(75)$ & & 73 & $28(38)$ & $45(62)$ & \\
\hline \multicolumn{9}{|l|}{ Tumor Size } \\
\hline$<2 \mathrm{~cm}$ & 21 & $12(57)$ & $12(57)$ & 0.612 & 23 & $11(48)$ & $12(52)$ & 0.341 \\
\hline$>2 \mathrm{~cm}$ & 73 & $47(64)$ & $47(64)$ & & 74 & $27(36)$ & $47(64)$ & \\
\hline \multicolumn{9}{|l|}{ Clinical Stage } \\
\hline $\mathrm{I}+\mathrm{II}$ & 48 & $31(65)$ & $31(65)$ & 0.831 & 63 & $32(51)$ & $31(49)$ & 0.002 \\
\hline III+IV & 46 & $28(61)$ & $28(61)$ & & 34 & $6(18)$ & $28(82)$ & \\
\hline \multicolumn{9}{|l|}{ Histological grade } \\
\hline $\mathrm{I}+\mathrm{II}$ & 63 & $33(52)$ & $33(52)$ & 0.003 & 56 & $23(41)$ & $33(59)$ & 0.689 \\
\hline $\mathrm{III}+\mathrm{IV}$ & 31 & $26(84)$ & $26(84)$ & & 41 & $15(37)$ & $26(63)$ & \\
\hline \multicolumn{9}{|l|}{ ER Status } \\
\hline Negative & 75 & $52(69)$ & $52(69)$ & 0.015 & 85 & $33(39)$ & $52(61)$ & 1 \\
\hline Positive & 19 & $7(37)$ & $7(37)$ & & 12 & $5(42)$ & $7(58)$ & \\
\hline \multicolumn{9}{|l|}{ PR Status } \\
\hline Negative & 70 & $46(66)$ & $46(66)$ & 0.337 & 73 & $27(37)$ & $46(63)$ & 0.477 \\
\hline Positive & 24 & $13(54)$ & $13(54)$ & & 24 & $11(46)$ & $13(54)$ & \\
\hline \multicolumn{9}{|l|}{ HER2 Status } \\
\hline Negative & 32 & $27(84)$ & $5(16)$ & 0.002 & 40 & $13(32)$ & $27(68)$ & 0.296 \\
\hline Positive & 62 & $32(52)$ & $30(48)$ & & 57 & $25(44)$ & $32(56)$ & \\
\hline \multicolumn{9}{|l|}{ TNBC } \\
\hline Negative & 61 & $31(5)$ & $30(49)$ & & 53 & $22(41)$ & $31(59)$ & 0.678 \\
\hline Positive & 33 & $28(85)$ & $5(15)$ & 0.002 & 44 & $16(36)$ & $28(64)$ & \\
\hline
\end{tabular}


Table 3. Statistical Relation between PTEN Gene Status and PTEN Protein Expression in Indian Breast Cancer

\begin{tabular}{|c|c|c|c|c|c|c|}
\hline \multirow[t]{2}{*}{ PTEN gene status } & \multirow[t]{2}{*}{ Total $(n=181)$} & \multicolumn{4}{|c|}{ PTEN Expression } & \multirow[t]{2}{*}{$\mathrm{p}$ Value } \\
\hline & & Negative & Low & Medium & High & \\
\hline Double hit Methylation $+\mathrm{LOH}$ & $46(25)$ & $32(70)$ & $12(26)$ & $2(4)$ & $0(0)$ & 0.037 \\
\hline Single hit (Methylation only) & $48(27)$ & $27(56)$ & $12(25)$ & $8(17)$ & $1(2)$ & \\
\hline Single hit LOH only & $52(29)$ & $30(58)$ & $21(40)$ & $1(2)$ & $0(0)$ & \\
\hline
\end{tabular}
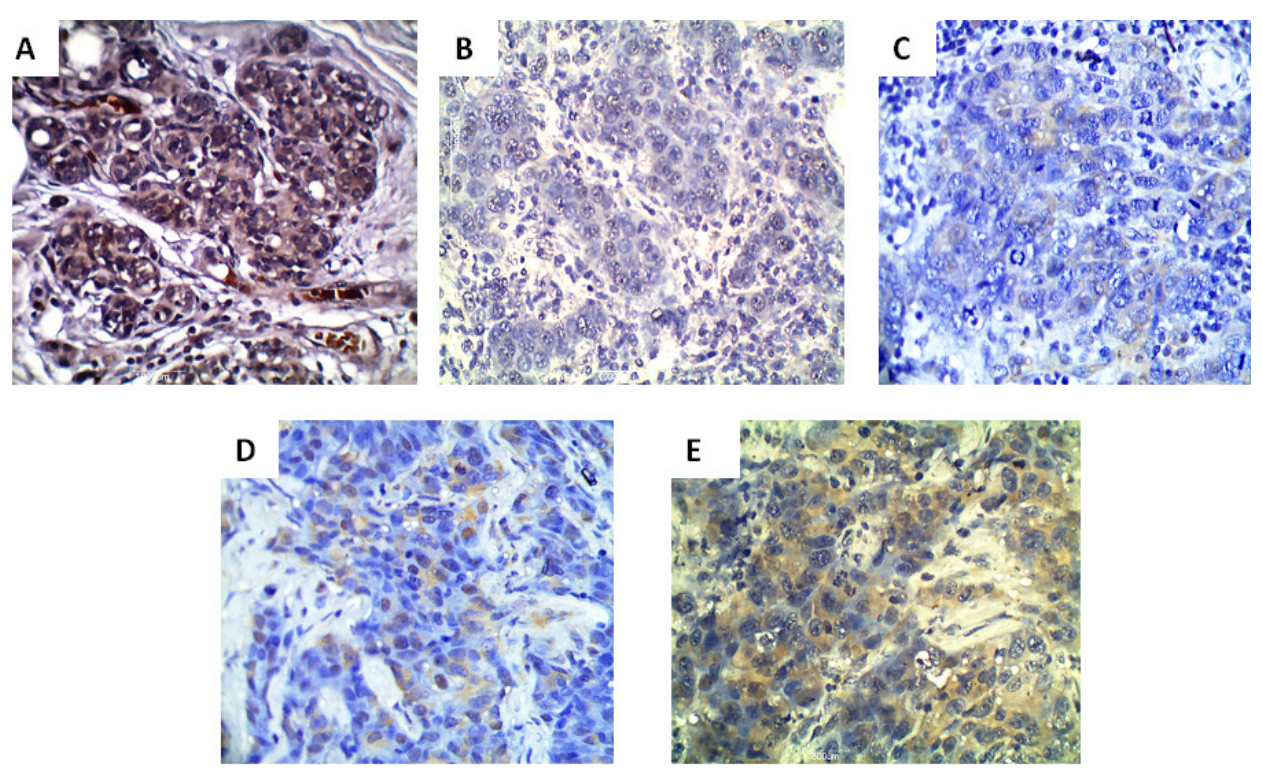

Figure 3. Representative Pictures of Immunostaining of PTEN Protein Expression in Human Breast Tissue Samples; (A) Normal breast tissue showing strong PTEN expression, (B) Breast cancer tissue showing negative expression of PTEN, (C) Breast cancer tissue showing low expression of PTEN protein.(D) \& (E) showing moderate to high expression of PTEN expression in breast cancer tissue. (magnification: 400X, scale bar $1000 \mu \mathrm{m}$ ). PTEN-positive staining is generally detected cytoplasmic signal that can easily be distinguished from PTEN-negative cases. In the presented example images, 3,3'-Diaminobenzidine (DAB) was used as a chromogen.

protein expression. Interestingly, a statistically significant relationship was found between the $\mathrm{LOH}$ and Loss of PTEN protein expression $[\mathrm{P}=<0.0001, P T E N$ negative (63\%) vs PTEN positive \{Low (34\%), Moderate(3\%), High $(0 \%)\}$ cases] as summarized in Table 1 . We also evaluated the correlation of PTEN expression with clinical variables in $\mathrm{LOH}$ positive cases and found significant correlations of PTEN loss with menopausal status, Her-2 negativity, and TNBC cases. On the contrary, in PTEN negative cases, LOH showed no correlation with any clinical variable (Supplementary Table 5).

PTEN promoter methylation and correlation with PTEN protein expression of breast cancer

Out of 181 sporadic breast cancer cases, promoter methylation of PTEN was observed in 52\% (94/181) cases. Moreover, PTEN promoter methylation was found to demonstrate a significant level of association with the PTEN protein loss $[\mathrm{P}=0.002$, PTEN negative $(63 \%)$ vs PTEN positive $\{$ Low (26\%), Moderate(11), High(1)\} cases]. Moreover, in PTEN negative cases level of promoter hypermethylation was higher than unmethylated cases (i.e., $63 \%$ vs $44 \%$ ) as illustrated in Table 1 . While correlating the PTEN expression in methylated cases, we observed a significant correlation of it with menopausal status, histological grade, ER status, Her-2 status and TNBC cases. In contrast, in PTEN negative cases, PTEN methylation showed a significant correlation only with clinical stage (Table 2).

\section{PTEN mutation screening}

The entire coding region of PTEN was screened. For each tumor and control samples, all nine exons were amplified. PCR products having altered mobility were directly sequenced. Based on our PTEN sequencing results, we found only $7 \%$ mutation in the north Indian population. Apparently, no significant correlation was found between the mutation and clinical variables.

PTEN expression status in methylation and LOH cases; two hit

There were $25 \%(46 / 181)$ cases in the present study, which harbored double hit (i.e, having both $\mathrm{LOH}$ and PTEN methylation). In these cases, 70\% (32/46) showed a dramatic loss of PTEN protein expression, thereby complete inactivation of PTEN gene as shown in Table 8. Although there were $26 \%(12 / 46)$ and $4 \%(2 / 46)$ cases which shown low and moderate expression of PTEN in spite of double hit (Table 3). This could be explained by an insufficient methylation at the promoter site and indicate that a certain level of methylation is needed for translational inactivation and silencing. We found that molecular events like LOH and methylation of PTEN gene play important role in breast cancer patient and have an immense impact on clinical variables. 
Table 4. Correlation of PTEN Protein Expression with TNBC Cases in a Subgroup Consists of Tumor Size and Menopausal Status

\begin{tabular}{|c|c|c|c|c|c|c|}
\hline \multirow[t]{2}{*}{ Menopausal Status } & \multirow[t]{2}{*}{ Tumor size } & \multirow[t]{2}{*}{ TNBC Status } & \multirow[t]{2}{*}{ Total } & \multicolumn{2}{|c|}{ PTEN Expression } & \multirow[t]{2}{*}{$\mathrm{p}$ Value } \\
\hline & & & & Negative & Positive & \\
\hline \multirow[t]{4}{*}{ Premenopausal } & $<2 \mathrm{~cm}$ & Negative & 15 & $5(33)$ & $10(68)$ & 1 \\
\hline & & Positve & 5 & $2(40)$ & $3(60)$ & \\
\hline & $>2 \mathrm{~cm}$ & Negative & 36 & $9(25)$ & $27(75)$ & 0.127 \\
\hline & & Positive & 17 & $8(47)$ & $9(53)$ & \\
\hline \multirow[t]{4}{*}{ Postmenopausal } & $<2 \mathrm{~cm}$ & Negative & 15 & $10(67)$ & $5(33)$ & 0.262 \\
\hline & & Positive & 6 & $6(100)$ & 0 & \\
\hline & $>2 \mathrm{~cm}$ & Negative & 57 & $29(51)$ & $28(49)$ & 0.0001 \\
\hline & & Positive & 30 & $28(93)$ & $2(7)$ & \\
\hline
\end{tabular}

Association of PTEN expression with clinical subgroups

Interestingly, we found a significant correlation between PTEN loss and menopausal status, which remains significant even in $\mathrm{LOH}$ positive and in PTEN methylated cases (Table 2) (Supplementary Table 5). To get a closer relationship, we further divided samples into premenopausal and postmenopausal cases. Where in premenopausal cases, we found a significant correlation of PTEN positive cases with clinical stage and histological grade while in postmenopausal cases, loss of PTEN expression showed significant correlation with advanced histological grade, ER negativity, Her-2 negativity and TNBC cases (Supplementary Table 6). To check the effect of tumor size, we also divided group in small and large tuomor sizes and found significant associations of PTEN with different variables (Supplementary Table 7). Finally, we examined the association of PTEN expression with TNBC cases in a combined group of menopausal status and Tumor size, where loss of PTEN expression was significantly correlated with the triple negative breast cancer of large tumor size in postmenopausal group (Table 3).

\section{Discussion}

PTEN aberration is frequently observed in human solid cancer, including breast cancer (Salmena et al., 2008). It is reported that PTEN demonstrates tumor suppression through the regulation of PIP3 in PI3K/ Akt pathway (Maehama and Dixon, 1998). In addition to genetic implication, the epigenetic crosstalk also plays a crucial role in breast cancer (Polyak, 2011). In the present study, we, therefore, investigated mutation, loss of heterozygosity, methylation status of the PTEN promoter region and their association with the expression level of PTEN protein. We further analyzed how different molecular aberrations of the PTEN gene are correlated with different clinicopathological parameters and define subgroups among north Indian breast cancer patients.

Through the immunostaining, we observed a $54 \%$ (97/181) loss of in PTEN protein expression as compared to normal controls which are in agreement with earlier studies reporting 33\% $-45 \%$ loss of PTEN protein in the breast cancers (Perren et al., 1999; Bose et al., 2002; Inanc et al., 2014). Notably, we found a significant relationship of PTEN loss with menopausal status, clinical stage, histological grade, Her-2 negativity and TNBC (triplenegative breast cancer). Interestingly, in the present study, $32 \%(58 / 181)$ cases were TNBC (triple-negative breast cancer) and out of which, 76\% (44/181) cases were also 'Quadruple Negative' (ER, PR, Her-2 and PTEN negative). Thus, we confirm PTEN loss a frequent event in Indian TNBC cases; that identified PTEN immunohistochemistry as an important tool in the TNBC group. In view of that PTEN loss may be a predictive marker for the treatment response.

Our study also speculates possible genetic and epigenetic factors involved in PTEN loss. We observed a comparatively higher $(54 \%) \mathrm{LOH}$ at PTEN region than previous studies (Singh et al., 1998; Rizvi et al., 2012; Feilotter et al., 1999), which was found to be significantly correlated with the loss of PTEN expression. Although, like other studies, we found very less $(\sim 7 \%)$ cases of mutation in Indian patient (Li et al., 1997; Tashiro et al., 1997; Guldberg et al., 1997). In addition, we noted 51.9\% (94/181) PTEN promoter methylation that was also significantly related to the loss of PTEN protein. This pattern is again supported by several pieces of evidence (Zhang et al., 2013; García et al., 2004). Our finding is congruent with an earlier study (Siddiqu et al., 2016) which have close pattern resemblance of PTEN expression and promoter methylation but their study lacks comprehensive illustration especially on the aspect of LOH which we have extensively evaluated in addition to the parameters taken by them (Siddiqui et al., 2016). In line with this, we also checked the combined effect (double hit) of PTEN methylation and LOH in PTEN loss. It is noteworthy, 25\% (46/181) cases showed concomitant LOH and PTEN promoter hypermethylation. Among these $25 \%$ samples, $70 \%(32 / 46)$ cases had complete expressional loss of PTEN. However, despite the double hit, we have found a low $26 \%(12 / 46)$ and medium $4 \%$ (2/46) expression of PTEN protein. The incomplete methylation at the promoter site could account for this low expression. Hence, our study provides an evidence that besides the double hit, both LOH and methylation of PTEN promoter are major the independent mechanism leading to loss of PTEN protein at least in north Indian population. 
To determine whether pathologic features can define a subset of women who are likely to have PTEN alterations, we correlated the molecular events with patients variables. Like an earlier study, we found that allelic loss at 10q23 region is more prevalent among patients less than 50 years of age, however, in contrast to the study, our result also underlines the implication of advanced clinical stage and ER positivity in Indian breast cancer patients (Garcia et al., 1999). We also found a significant role of PTEN methylation in tumor progression. In addition, loss of PTEN expression has shown a strong relationship with menopausal status and TNBC even after grouping in LOH positive and PTEN methylation subgroups. Our study also found that significantly correlated Quadruple group (TNBC+PTEN negative) is predominantly found in tumors of the large size of a postmenopausal subset.

To the best of our knowledge, this is the first study reporting implication of the combinational frequency of methylation and $\mathrm{LOH}$ at the PTEN gene region with the loss of $P T E N$ protein in north Indian breast cancer patients. We are also among the first to evaluate PTEN protein expression status in defining clinical subgroups of Indian breast cancer patients. Our study defines a quadruple negative group with distinct features, hence, suggesting that more effective therapeutic strategies should be developed in TNBCs by considering these prognostic factors together in north Indian breast cancer patients.

In summary, our data suggest that along with the double hit, PTEN methylation and $\mathrm{LOH}$ are the independent mechanism of PTEN inactivation in a subset of Indian patients. We also suggest that a biological classification based on PTEN expression profile can truly enrich the targets in breast cancer. To boot, PTEN expression analysis for Indian breast cancer patients may be used as a cost-effective approach in distinguishing the more favorable group for a particular treatment.

\section{Conflict of interest}

All authors declare that they do not have any conflicts of interest.

\section{Acknowledgments}

This work was supported by grants from the DST (Department of science and technology) and UGC (University grants commission).

\section{References}

Ali A, Saluja SS, Hajela K, Mishra PK, Rizvi MA (2014). Mutational and expressional analyses of PTEN gene in colorectal cancer from Northern India. Mol Carcinog, 53, $45-52$.

Anonymous (2009). Breast cancer in developing countries. Lancet (London, England), 374, 1567.

Bose S, Crane A, Hibshoosh H, et al (2002). Reduced expression of PTEN correlates with breast cancer progression. Hum Pathol, 33, 405-9.

Feilotter HE, Coulon V, McVeigh JL, et al (1999). Analysis of the $10 \mathrm{q} 23$ chromosomal region and the PTEN gene in human sporadic breast carcinoma. Br J Cancer, 79, 718-23.

Garcia JM, Silva JM, Dominguez G, et al (1999). Allelic loss of the PTEN region (10q23) in breast carcinomas of poor pathophenotype. Breast Cancer Res Treat, 57, 237-43.

García JM, Silva J, Peña C, et al (2004). Promoter methylation of the PTEN gene is a common molecular change in breast cancer. Genes Chromosomes Cancer, 41, 117-24.

Guldberg P, Thor Straten P, Birck A, et al (1997). Disruption of the MMAC1/PTEN gene by deletion or mutation is a frequent event in malignant melanoma. Cancer Res, 57, 3660-3.

Hanahan D, Weinberg RA(2011). Hallmarks of cancer: The next generation. Cell, 144, 646-74.

Inanc M, Ozkan M, Karaca H, et al (2014). Cytokeratin 5/6, c-Met expressions, and PTEN loss prognostic indicators in triple-negative breast cancer. Med Oncol, 31, 801.

Jemal A, Bray F, Center MM, et al (2011). Global cancer statistics. CA Cancer J Clin, 61, 69-90.

Li J, Yen C, Liaw D, et al (1997). PTEN, a putative protein tyrosine phosphatase gene mutated in human brain, breast, and prostate cancer. Science (New York, N.Y.), 275, 1943-7.

Lu Y, Lin Y-Z, LaPushin R, et al (1999). The PTEN/MMAC1/TEP tumor suppressor gene decreases cell growth and induces apoptosis and anoikis in breast cancer cells. Oncogene, 18, 7034-45.

Lynch ED, Ostermeyer EA, Lee MK, et al (1997). Inherited mutations in PTEN that are associated with breast cancer, Cowden disease, and Juvenile Polyposis. Am J Hum Genet, 61, 1254-60.

Maehama T, Dixon JE (1998). The tumor suppressor, PTEN/ MMAC1, dephosphorylates the lipid second messenger, phosphatidylinositol 3,4,5-trisphosphate. J Biol Chem, 273, 13375-8.

Nelen MR, Kremer H, Konings IB, et al (1999). Novel PTEN mutations in patients with Cowden disease: absence of clear genotype-phenotype correlations. Eur J Hum Genet, 7, 267-73.

Perren A, Weng L-P, Boag AH, et al (1999). Immunohistochemical evidence of loss of PTEN expression in primary ductal adenocarcinomas of the breast. Am J Pathol, 155, 1253-60.

Polyak K (2011). Heterogeneity in breast cancer. J Clin Invest, 121, 3786-8.

Rizvi MMA, Alam MS, Ali A, et al (2011). Aberrant promoter methylation and inactivation of PTEN gene in cervical carcinoma from Indian population. J Cancer Res Clin Oncol, 137, 1255-62.

Rizvi MMA, Alam MS, Mehdi SJ, Ali A, Batra S (2012). Allelic loss of 10q23.3, the PTEN gene locus in cervical carcinoma from Northern Indian population. Pathol Oncol Res, 18, 309-13.

Salmena L, Carracedo A, Pandolfi PP (2008). Tenets of PTEN tumor suppression. Cell, 133, 403-14.

Shetty PJ, Pasupuleti N, Chava S, Nasaruddin K, Hasan Q (2011). Altered transcription and expression of PTEN in breast tumors: Is it regulated by hypermethylation?. Breast Dis, 33, 27-33.

Siddiqui S, Akhter N, Deo SVS, Shukla NK, Husain SA (2016). A study on promoter methylation of PTEN in sporadic breast cancer patients from North India. Breast Cancer, 15, 1-10.

Singh B, Ittmann MM, Krolewski JJ (1998). Sporadic breast cancers exhibit loss of heterozygosity on chromosome segment 10q23 close to the Cowden disease locus. Genes Chromosomes Cancer, 21, 166-71.

Song MS, Salmena L, Pandolfi PP (2012). The functions and regulation of the PTEN tumour suppressor. Nat Rev Mol Cell Biol, 13, 283-96.

Steck PA, Pershouse MA, Jasser SA, et al (1997). Identification of a candidate tumour suppressor gene, MMAC1, at chromosome 10q23.3 that is mutated in multiple advanced cancers. Nat Genet, 15, 356-62. 
Tamura M, Gu J, Matsumoto K, et al (1998). Inhibition of cell migration, spreading, and focal adhesions by tumor suppressor PTEN. Science (New York, N.Y.), 280, 1614-7.

Tashiro H, Blazes MS, Wu R, et al (1997). Mutations in PTEN are frequent in endometrial carcinoma but rare in other common gynecological malignancies. Cancer Res, 57, 3935-40.

Vivanco I, Sawyers CL (2002). The phosphatidylinositol 3-Kinase-AKT pathway in human cancer. Nat Rev Cancer, 2, 489-501.

Wahabi K, Perwez A, Rizvi MA (2018). Parkin in Parkinson's disease and cancer: a double-edged sword. Mol Neurobiol, http://doi.org/10.1007/s12035-018-0879-1.

Zhang H-Y, Liang F, Jia Z-L, Song S-T, Jiang Z-F (2013). PTEN mutation, methylation and expression in breast cancer patients. Oncol Lett, 6, 161-8.

\section{๑๐ब}

This work is licensed under a Creative Commons AttributionNon Commercial 4.0 International License. 